Supporting Information

\title{
Copper-Mediated DNA-Scaffolded Silver Nanocluster on-off Switch for Detection of Pyrophosphate and Alkaline Phosphatase
}

\author{
Jin-Liang Ma ${ }^{\dagger}$, Bin-Cheng Yin*, ${ }^{*}$, Xin $\mathrm{Wu}^{\ddagger}$, and Bang-Ce Ye*, ${ }^{* \xi}$ \\ ${ }^{\dagger}$ Lab of Biosystem and Microanalysis, State Key Laboratory of Bioreactor Engineering, East \\ China University of Science \& Technology, Shanghai, 200237, China \\ ${ }^{\xi}$ School of Chemistry and Chemical Engineering, Shihezi University, Xinjiang, 832000, China \\ ${ }^{\ddagger}$ Department of Rheumatology and Immunology, Shanghai Changzheng Hospital, The Second \\ Military Medical University, Shanghai, 200433, China
}

Corresponding author: Bin-Cheng Yin, binchengyin@ecust.edu.cn; Bang-Ce Ye, bcye@ecust.edu.cn, Fax no. 0086-21-64252094

\section{Table of content:}

1. The morphology characteristics of AgNCs (Figure S1)

2. The feasibility of the switch for PPi and ALP detection (Figure S2-S4)

3. The optimization of the method for PPi detection (Figure S5-S7)

4. Comparison of our method and other nanomaterial-based methods (Table S1)

5. The performance of the reaction system for detecting PPi and ALP spiked in $1 \%$ diluted bovine serum (Figure S8-S9 and Table S2)

6. The performance of the method for sensing PPi in synovial fluid and ALP in human serum (Figure S10-S13 and Table S3-S4)

7. References 


\section{The morphology characteristics of AgNCs}
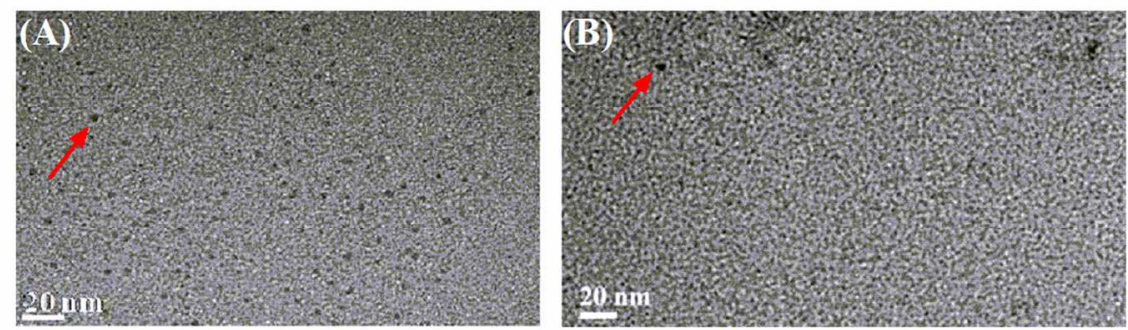

Figure S1. TEM images of DNA/AgNCs from the reaction system before (A) and (B) after adding $\mathrm{Cu}^{2+}$. 


\section{The feasibility of the switch for PPi and ALP detection}

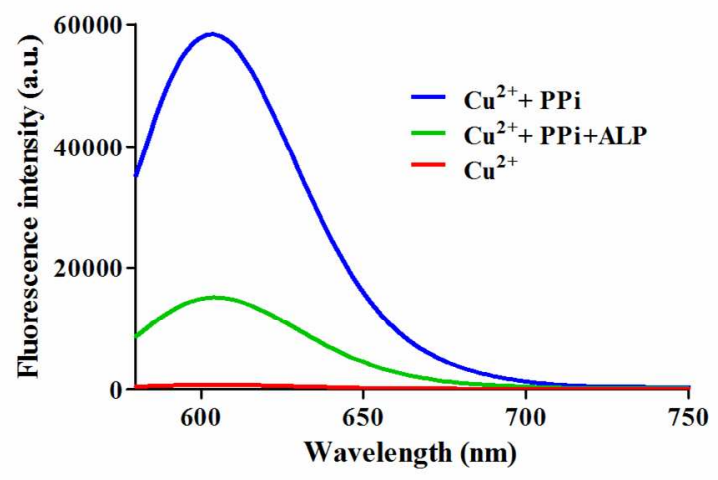

Figure S2. Fluorescence emission spectra of DNA/AgNCs after adding $\mathrm{Cu}^{2+}$ (red line), $\mathrm{Cu}^{2+}+\mathrm{PPi}$ (blue line), and $\mathrm{Cu}^{2+}+\mathrm{PPi}+\mathrm{ALP}$ (green line).

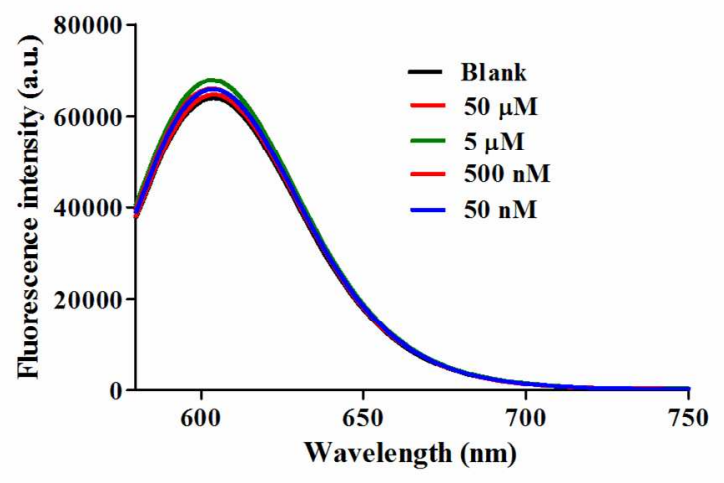

Figure S3. Fluorescence emission spectra of DNA/AgNCs after adding PPi only. 


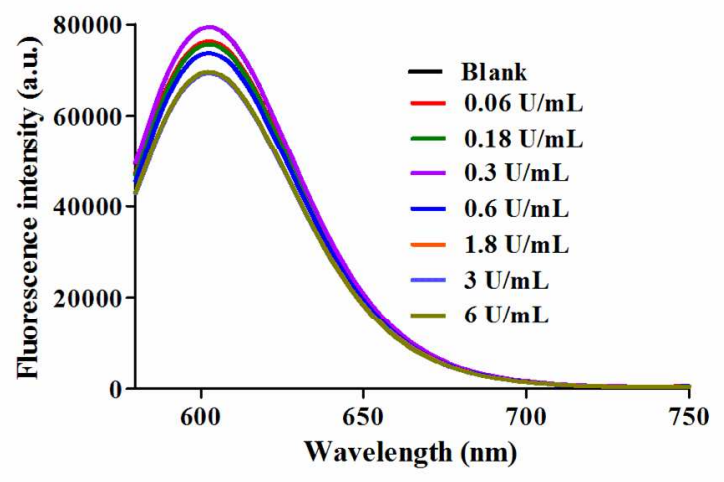

Figure S4. Fluorescence emission spectra of DNA/AgNCs after adding ALP only. 


\section{The optimization of the method for PPi detection}

(A)

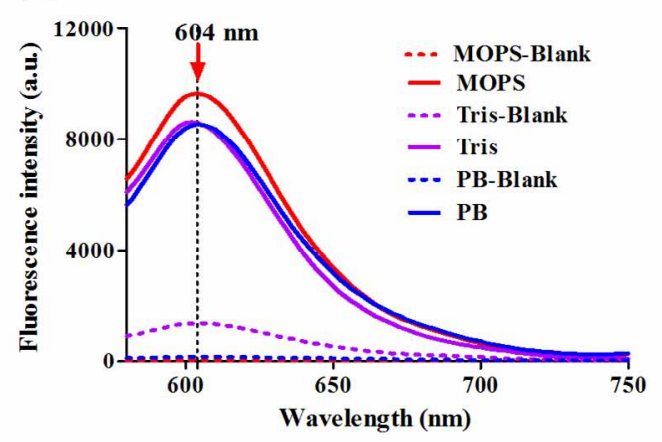

(B)

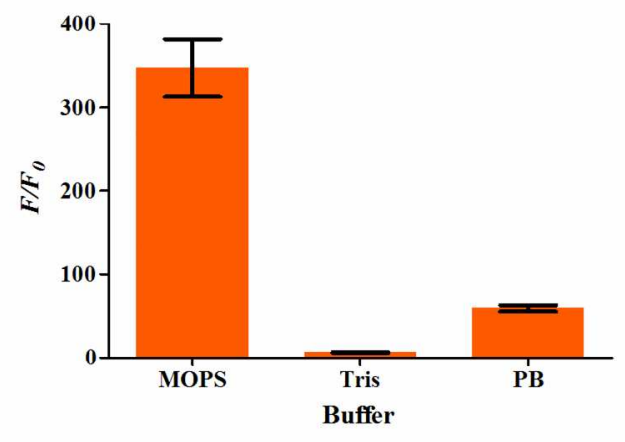

Figure S5. The selection of buffer used in the proposed AgNC on-off switch for detection of PPi. (A) Fluorescence emission responses of the proposed system obtained in three buffers using 5 $\mu \mathrm{M} \mathrm{Cu}{ }^{2+}$ in the absence and presence of $50 \mu \mathrm{M}$ PPi: $10 \times$ MOPS $(200 \mathrm{mM}$ MOPS, $500 \mathrm{mM}$ $\mathrm{NaNO}_{3}, \mathrm{pH} 7.0$ ), $10 \times$ Tris buffer $(200 \mathrm{mM}$ Tris, $500 \mathrm{mM} \mathrm{NaNO} 3, \mathrm{pH} 7.4$ ), $10 \times \mathrm{PB}$ (phosphate buffer) (38 mM NaH $2 \mathrm{PO}_{4}, 62 \mathrm{mM} \mathrm{Na} \mathrm{HPO}_{4}, 500 \mathrm{mM} \mathrm{NaNO}$, pH 7.0). (B) Bar graph of fluorescence ratio $\left(F / F_{0}\right)$ responses to the different buffers. $F$ and $F_{0}$ are the fluorescence intensities at a peak value of $604 \mathrm{~nm}$ in the presence and absence of PPi, respectively. The error bars were calculated from three independent experiments. 
(A)

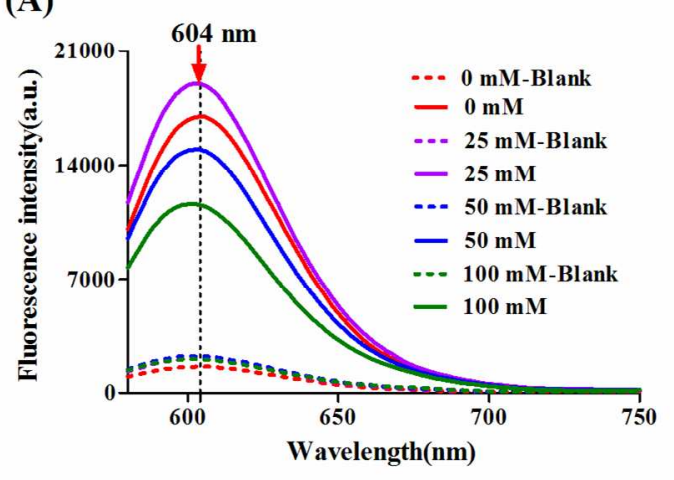

(B)

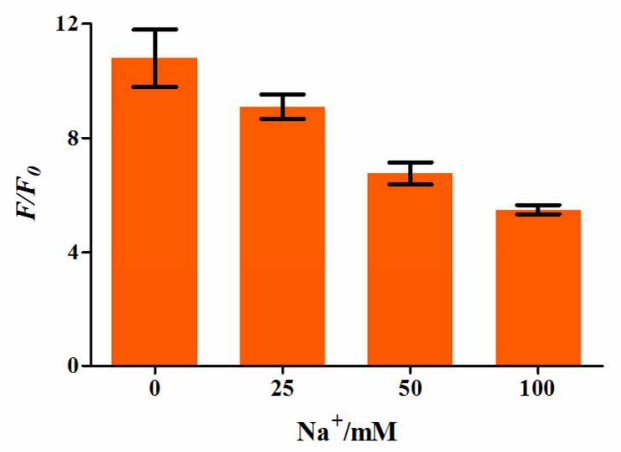

Figure S6. The effect of $\mathrm{Na}^{+}$to the performance of the proposed AgNC on-off switch for detection of PPi. (A) Fluorescence emission spectra responses of the proposed system carried out after adding $\mathrm{Na}^{+}$with different concentration. (B) Bar graph of fluorescence ratio $\left(F / F_{0}\right)$ responses to the different concentration of $\mathrm{Na}^{+} . F$ and $F_{0}$ are the fluorescence intensities at a peak value of $604 \mathrm{~nm}$ in the presence and absence of PPi, respectively. The error bars were calculated from three independent experiments. 
(A)

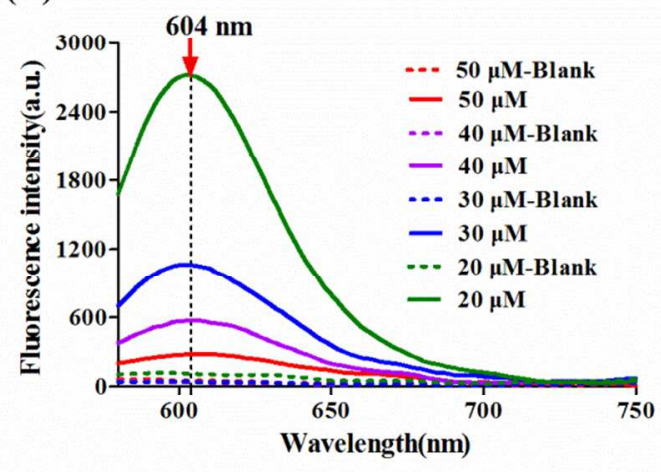

(B)

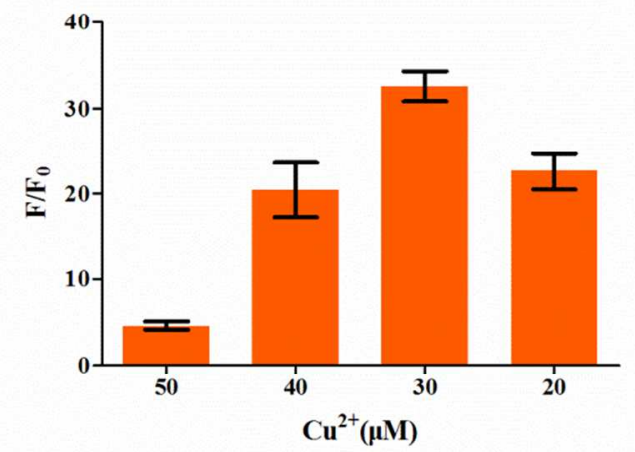

Figure S7. Optimization of $\mathrm{Cu}^{2+}$ concentration in the reaction system carried out in $1 \%$ diluted bovine serum. (A) The fluorescence spectra of the reaction system containing $\mathrm{Cu}^{2+}$ with different concentration and $100 \mu \mathrm{M}$ PPi. (B) Relative fluorescence intensity $\left(F / F_{0}\right)$ responses to the different concentrations of $\mathrm{Cu}^{2+} . F$ and $F_{0}$ are the fluorescence intensities at $604 \mathrm{~nm}$ in the presence and absence of PPi, respectively. The error bars were calculated from three independent experiments. 


\section{Comparison of our method and other nanomaterial-based methods}

Table S1. Comparison of sensitivity of our method and other nanomaterial-based methods for PPi and ALP detection

\begin{tabular}{cccc}
\hline Nanomaterial & Analyte & Detection limit & Reference \\
\hline Carbon quantum dot & PPi & $2.56 \mu \mathrm{M}$ & 1 \\
Gold nanoparticle & PPi & $130 \mathrm{nM}$ & 3 \\
Gold nanocluser & PPi & $1 \mu \mathrm{M}$ & Our method \\
AgNCs & PPi & $112.69 \mathrm{nM}$ & 4 \\
Copper nanoparticle & ALP & $0.3 \mathrm{U} / \mathrm{L}$ & 5 \\
Cu/AgNCs & ALP & $0.3 \mathrm{U} / \mathrm{L}$ & 6 \\
Carbon quantum dot & ALP & $1.4 \mathrm{U} / \mathrm{L}$ & 7 \\
Carbon quantum dot & ALP & $1.1 \mathrm{U} / \mathrm{L}$ & Our method \\
AgNCs & ALP & $5 \mathrm{U} / \mathrm{L}$ & \\
\hline
\end{tabular}


5. The performance of the reaction system for detecting PPi and ALP spiked in $1 \%$ diluted bovine serum

(A)

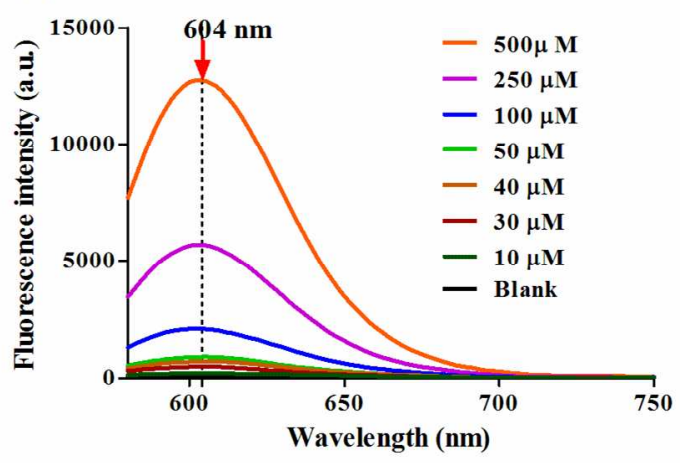

(B)

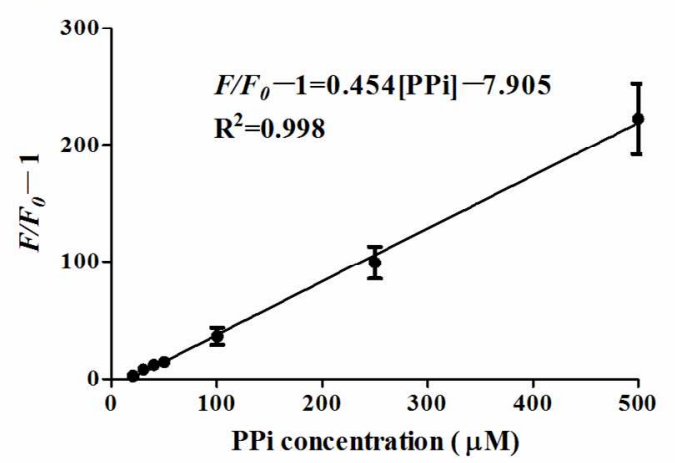

Figure S8. The performance of the proposed method applied in 1\% diluted bovine serum. (A) The fluorescence spectra of the reaction system after adding PPi with different concentration. (B) Plot of the linear relationship between the fluorescence ratio $\left(F / F_{0}-1\right)$ and the concentration of PPi. The error bars were calculated from three independent experiments.

Table S2. Recovery results of ALP in 1\% diluted bovine serum at three concentrations

\begin{tabular}{|c|c|c|c|}
\hline Spiked amount (U/mL) & Detected amount (U/mL) & Recovery (\%) & CV (\%) \\
\hline 0.2 & 0.233 & 116.40 & 6.32 \\
\hline 0.3 & 0.309 & 103.07 & 6.13 \\
\hline 0.4 & 0.383 & 95.65 & 1.35 \\
\hline
\end{tabular}



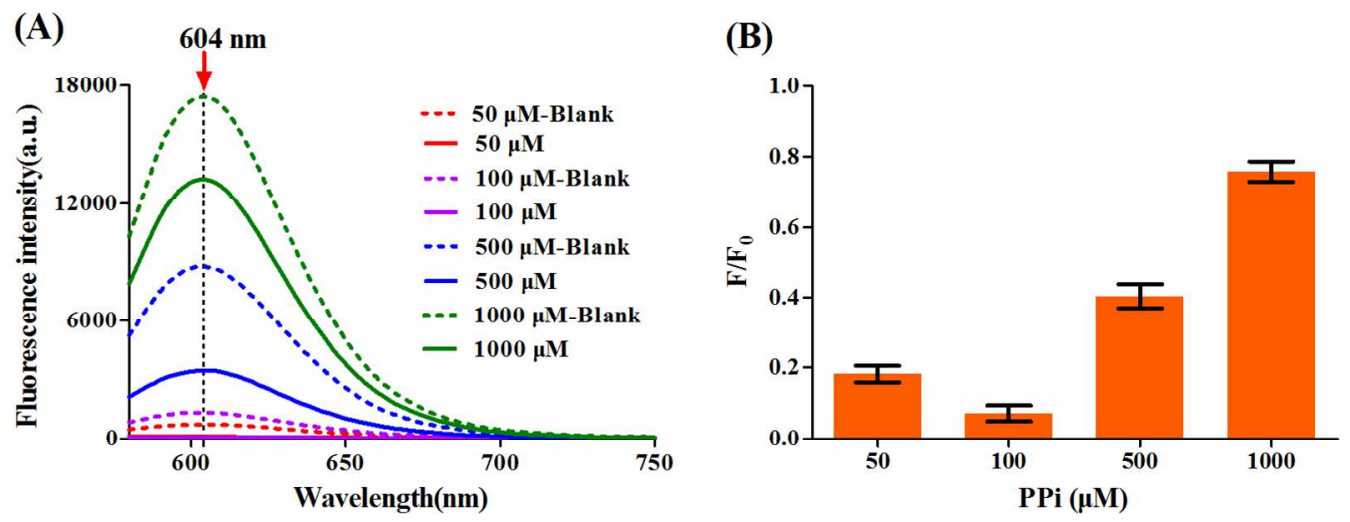

Figure S9. Optimization of PPi concentration in the reaction system for ALP detection carried out in $1 \%$ diluted bovine serum. (A) The fluorescence spectra of the reaction system containing PPi with different concentration, $30 \mu \mathrm{M} \mathrm{Cu}^{2+}$ and $1 \mathrm{U} / \mathrm{mL}$ ALP. (B) Relative fluorescence intensity $\left(F / F_{0}\right)$ responses to the different concentrations of PPi. $F$ and $F_{0}$ are the fluorescence intensities at $604 \mathrm{~nm}$ in the presence and absence of ALP, respectively. The error bars were calculated from three independent experiments. 


\section{The performance of the method for sensing PPi in synovial fluid and ALP in human serum}

(A)

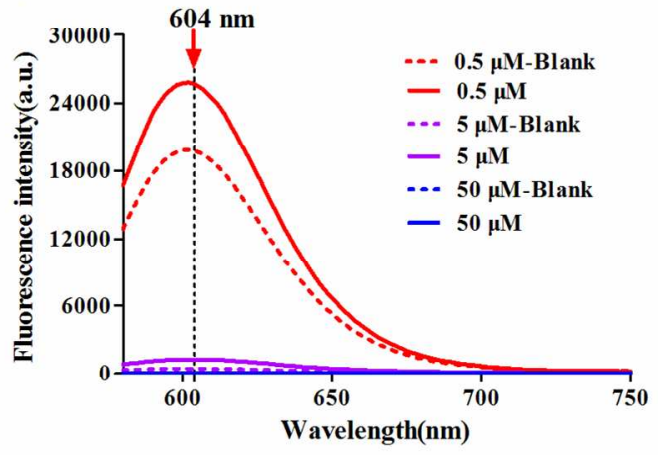

(B)

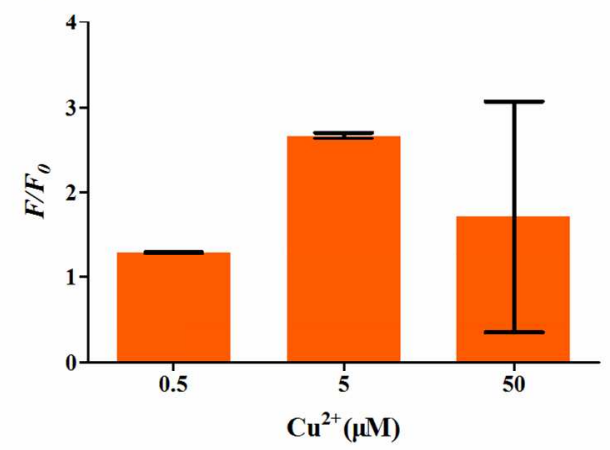

Figure S10. Optimization of $\mathrm{Cu}^{2+}$ concentration in the reaction system carried out in synovial fluid from arthritis patient and healthy control. (A) The fluorescence spectra of the reaction system containing $\mathrm{Cu}^{2+}$ with different concentration. (B) Relative fluorescence intensity $\left(F / F_{0}\right)$ responses to the different concentrations of $\mathrm{Cu}^{2+} . F$ and $F_{0}$ are the fluorescence intensities at 604 $\mathrm{nm}$ of the reaction system for PPi detection in synovial fluid from arthritis patient and healthy control, respectively.

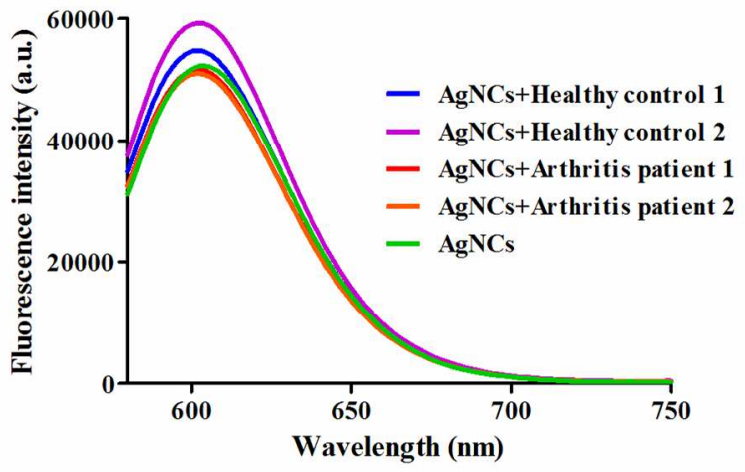

Figure S11. The effect of synovial fluid from arthritis patient and healthy controls to the synthesis of silver nanoclusters without addition of $\mathrm{Cu}^{2+}$. 


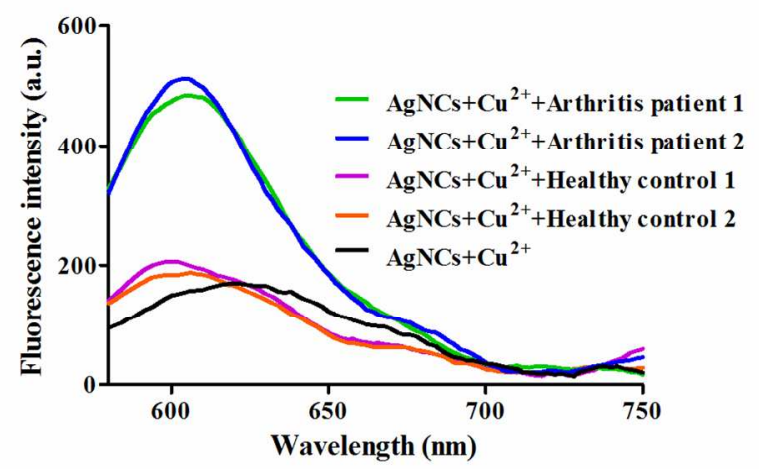

Figure S12. The fluorescence spectra of the reaction system carried out in synovial fluid from arthritis patients and healthy controls.

Table S3. The determination of PPi in synovial fluid

\begin{tabular}{cccc}
\hline Analytical methods & Arthritis patients & PPi $(\boldsymbol{\mu M})$ & References \\
\hline Our methods & No. 1 & $34.74 \pm 12.02$ & $/$ \\
& No. 2 & $30.92 \pm 13.08$ & $/$ \\
Colorimetric method & $/$ & $23.5 \pm 5.3$ & 8 \\
Enzymatic analytical method & $/$ & $23.9 \pm 10.5$ & 9 \\
$\mathrm{P}^{32}$ chromatographic method & $/$ & $22.7 \pm 13.8$ & 9 \\
\hline
\end{tabular}




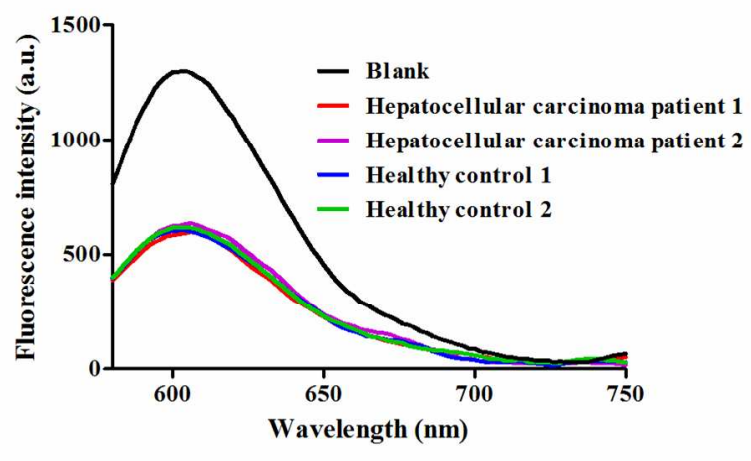

Figure S13. The fluorescence spectra of the reaction system carried out in human serum from hepatocellular carcinoma patients and healthy controls.

Table S4. The determination of ALP in human serum

\begin{tabular}{ccc}
\hline Samples & Number & ALP (U/mL) \\
\hline Hepatocellular carcinoma patients & No. 1 & $0.201 \pm 0.053$ \\
Healthy controls & No. 2 & $0.184 \pm 0.058$ \\
& No. 1 & $0.192 \pm 0.010$ \\
& No. 2 & $0.196 \pm 0.003$ \\
\hline
\end{tabular}




\section{References}

(1) Qian, Z.; Chai, L.; Zhou, Q.; Huang, Y.; Tang, C.; Chen, J.; Feng, H. Anal. Chem. 2015, 87, 7332-7339.

(2) Deng, J.; Yu, P.; Yang, L.; Mao, L. Anal. Chem. 2013, 85, 2516-2522.

(3) Sun, J.; Yang, F.; Zhao, D.; Yang, X. Anal. Chem. 2014, 86, 7883-7889.

(4) Zhang, L.; Zhao, J.; Duan, M.; Zhang, H.; Jiang, J.; Yu, R. Anal. Chem. 2013, 85, 3797-3801.

(5) He, Z.; Ye, T.; Li, C.; Su, C.; Ji, X. RSC Adv. 2015, 5, 55336-55339.

(6) Qian, Z.; Chai, L.; Tang, C.; Huang, Y.; Chen, J.; Feng, H. Anal. Chem. 2015, 87, 2966-2973.

(7) Qian, Z. S.; Chai, L. J.; Huang, Y. Y.; Tang, C.; Shen, J. J.; Chen, J. R.; Feng, H. Biosens. Bioelectron. 2015, 68, 675-680.

(8) Medicine, O. E. M. I. Arthritis Rheum.1973, 16, 171-178.

(9) Russell, R. G. G.; Bisaz, S.; Fleisch, H.; Currey, H. L. F.; Rubinstein, H. M.; Dietz, A. A.; Boussina, I.; Micheli, A.; Fallet, G. Lancet 1970, 2, 899-902. 